\section{CJD without borders}

\section{José Eriton G. Cunha (1), Jessica C. F. Nascimento and João R. M. Oliveira}

In their recent Review, Watson et al. discussed the need for ongoing international surveillance of Creutzfeldt-Jakob Disease (CJD) (Watson, N. et al. The importance of ongoing international surveillance for Creutzfeldt-Jakob disease. Nat. Rev. Neurol. 17, 362-379 $\left.(2021)^{1}\right)$. CJD belongs to a unique group of rare neurodegenerative pathologies called prion diseases for which, to date, no cure or effective treatment is available. Despite being rare, CJD is a great concern to public health, particularly its variant (vCJD) and iatrogenic (iCJD) forms, which have a long incubation period ${ }^{2}$.

We agree with Watson et al. ${ }^{1}$ that reference centres and international collaboration are crucial for improved management and discussion of rare diseases such as CJD. This kind of collaboration is pivotal to help countries with a deficient or nonexistent surveillance system to manage cases with greater accuracy and efficiency. For example, in Brazil, a collaboration between the Ministry of Health and several research groups was established in the early 2000s and resulted in a protocol for notification, follow-up and management of reported cases of CJD that is still in use today ${ }^{3}$. However, in 2018, some of these collaborations were discontinued and since then genetic assessment has not been performed in individuals with suspected CJD, worsening an already weak surveillance system $^{4}$. Although the Brazilian CJD protocol involves a simple but well-designed report sheet, accessing the resulting epidemiological data is not straightforward. The data must be requested from the Brazilian Ministry of Health online. When provided, the data are codified in almost all fields. When we compared data obtained via this route with a Ministry of Health publication covering the same time period $^{5}$, we identified some differences in the number of cases reported, highlighting the challenges involved in collecting and sharing accurate data on rare diseases.

Recently, after an extensive effort to access and decode Brazilian epidemiological data and make it more accessible to the population, our group launched an online dashboard called DCJ EpiShiny, to map and share information about CJD in Brazil (in Portuguese). We also created CJD Without Borders, an international databank based on the databank of the CJD International Surveillance Network (CJDISN), formerly EuroCJD, but with additional data from Asian, South American and African countries. The main goal of CJD Without Borders is to pool information on CJD cases from around the world and compile information about geographical distribution and clinicalpathological features. Unfortunately, to date, we have been unable to include data from African countries as we face limitations in data availability.

The recommendation of Watson et al. ${ }^{1}$ that countries with established CJD surveillance systems should provide support to other countries is outstanding. We support this initiative and encourage actions towards this aim. The CJDISN is an exceptional example of how we can create a unified source of global CJD data. By using the CJDISN as a model and expanding the collaborations to additional countries, we would be able to improve our understanding of the epidemiology and clinical features of CJD. Such an international effort could also facilitate the exchange of biological samples between centres, accelerating CJD research and clinical trials.

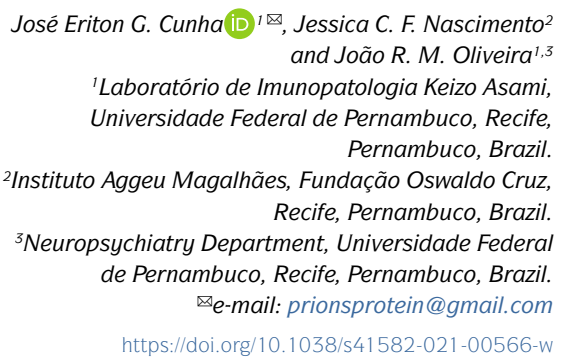

José Eriton G. Cunha iD ${ }^{1 凶}$, Jessica C. F. Nascimento ${ }^{2}$ and João R. M. Oliveira ${ }^{1,3}$ 'Laboratório de Imunopatologia Keizo Asami, Universidade Federal de Pernambuco, Recife, Pernambuco, Brazil. 2Instituto Aggeu Magalhães, Fundação Oswaldo Cruz, Recife, Pernambuco, Brazil. ${ }^{3}$ Neuropsychiatry Department, Universidade Federal de Pernambuco, Recife, Pernambuco, Brazil. 凶e-mail:prionsprotein@gmail.com https://doi.org/10.1038/s41582-021-00566-w

1. Watson, N. et al. The importance of ongoing international surveillance for Creutzfeldt-Jakob disease. Nat. Rev. Neurol. 17, 362-379 (2021).

2. Tee, B. L., Longoria Ibarrola, E. M. \& Geschwind, M. D. Prion diseases. Neurol. Clin. 36, 865-897 (2018).

3. Martins, V. R. et al. Prion diseases are under compulsory notification in Brazil. Surveillance of cases evaluated by biochemical and/or genetic markers from 2005 to 2007. Dement. Neuropsychol. 1, 347-355 (2007).

4. Cunha, J. E. G. da \& Oliveira, J. R. M. Compulsory notification of prion diseases in Brazil: what has changed since 2005? Dement. Neuropsychol. 2 , 155-156 (2008).

5. Situação epidemiológica dos casos da doença de Creutzfeldt-Jakob, Brasil, 2005 a 2013 [Portuguese] Secr. Vigilância em Saúde - Ministério da Saúde 2. 35-42 (2016)

Acknowledgements

The authors acknowledge Terri Lindsay from National CJD Research \& Surveillance Unit (United Kingdom) for contributing to CJD without Borders Web App and the Brazilian Ministry of Health for providing the data for the DCJ EpiShiny Web App. They also thank Coordenação de Aperfeiçoamento de Pessoal de Nivel Superior (CAPES) and Conselho Nacional de Desenvolvimento Científico e Tecnológico (CNPq) for the financial support.

\section{Competing interests}

The authors declare no competing interests.

\section{RELATED LINKS}

CJD International Surveillance Network (CJDISN):

https://www.eurocjd.ed.ac.uk/

CJD Without Borders: https://epicjd.shinyapps.io/cjdMAP/

DCJ Epishiny: https://epicjd.shinyapps.io/dcjBRASIL/ 